\title{
LATERAL LAMINATION APPROACH FOR MULTILAYER PIEZOELECTRIC MICROACTUATOR
}

\author{
Xiaosong Wu ${ }^{1}$, Guang Yuan ${ }^{2}$, Seong-O Choi ${ }^{2}$, Yanzhu Zhao ${ }^{2}$, \\ Seong-Hyok Kim ${ }^{2}$, Yong-Kyu Yoon ${ }^{2}$ and Mark G. Allen ${ }^{2}$ \\ Schools of Polymer, Textile and Fiber Engineering ${ }^{1}$ and Electrical and Computer Engineering ${ }^{2}$ \\ 791 Atlantic Drive, N.W., Georgia Institute of Technology \\ Atlanta, GA, 30332, USA
}

\begin{abstract}
This paper reports a simple lateral lamination scheme for the fabrication of multilayer piezoelectric actuators. The fabrication scheme consists of dicing of a high- $\mathrm{d}_{33}$ piezoelectric coefficient lead zirconate titanate (PZT) material, photolithographic highaspect-ratio SU-8 definition of electrical isolation, and micromolding of conductive polymer electrodes. Backside and inclined UV exposure has been exploited to secure the $1 \mathrm{~mm}$-thick SU-8 pillar definition of internal electrodes and to prevent the formation of a tapered SU-8 pillar shape that allows electrical short paths. An electrically conductive polymer composite (ECPC, silver-PMMA: $40 \mathrm{vol} \% \mathrm{Ag}$ ) has been utilized for making moldable electrodes. In fabrication and actuation, it is advantageous to have the isolation structures and the electrodes to be both made with polymeric materials so that they have similar thermal and mechanical properties. An 8-layer device is successfully fabricated and tested. A displacement of 0.63 micron is achieved at $100 \mathrm{~V}$ driving voltage, which agrees well with finite element simulation results.
\end{abstract}

\section{INTRODUCTION}

Piezoelectric actuators are used in a number of applications such as precision positioners for metrology and interferometry, ultrasonic transducers, and scanning microscopes [1-3]. The miniaturization of these and other piezoelectric components is important for the development of compact, low-driving voltage, and cost-effective devices. In order to have reasonable displacement at low voltage, often a multilayer piezoelectric scheme is utilized. Compared to their single layer counterparts, multilayer piezoelectric actuators offer several advantages such as low driving voltages while maintaining high electric fields necessary for actuation, rapid response time, and high electromechanical coupling [4]. A typical lamination scheme (Fig 1a) relies on a vertical stack of alternating layers of electrodes and piezoelectric layers, where each lamination step requires electrodes to be deposited and patterned or aligned [2, 3]. Alternatively, a planar fabrication method can be utilized to form a lateral multilayer piezoelectric actuator, in which a series of vertical grooves are cut into a PZT crystal and metallized with appropriate isolation to form a lateral multilayer structure. Such a structure was demonstrated using electroplating of electrodes followed by laser-assisted etching of PZT and electrodes [5]. However, process challenges of electroplating in deep grooves as well as issues with the laser assisted etching were also reported.

To avoid these difficulties, we propose an alternative, simple, and cost-effective lateral lamination scheme (Fig 1b) for a multilayered piezoelectric actuator. The process involves PZT dicing, photolithographic definition of high-aspect-ratio SU-8 pillars for electrical isolation, and micromolding of ECPC electrodes. This approach overcomes the reported process difficulties of [5], while simultaneously maintaining the advantages of multilayer piezoelectric actuators.

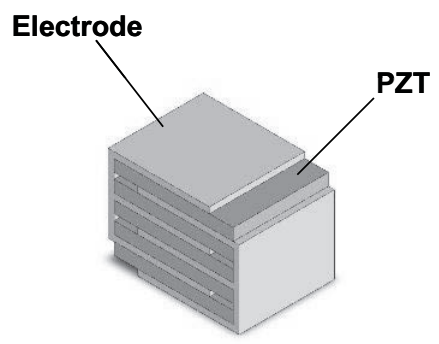

(a)

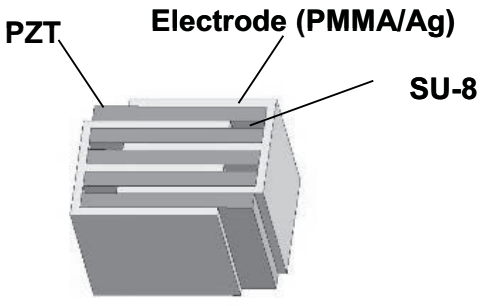

(b)

Figure 1. Lamination architectures for multilayer piezoelectric actuator: (a) conventional vertical stack lamination, (b) proposed lateral lamination

In this study, an effective photolithographic patterning scheme for the $1 \mathrm{~mm}$-thick isolation structure has been demonstrated. Specific advantages associated with the molding of internal electrodes include: (1) the ECPC casting through highaspect-ratio trench is time-effective, compared to the long processing time of an electroplating approach; (2) the ECPC electrodes alleviate void formation issues which may occur during the plating through the deep trenches; (3) the silver-PMMA composite has mechanical and thermal properties similar to those of the isolation SU-8, which increases the stability of the actuator. Using these schemes, the PZT actuator has been designed, tested and characterized.

\section{FABRICATION}

Figure 2 illustrates the fabrication process of PZT multilayer actuators. The $1 \mathrm{~mm}$-thick PZT plate used in this study is a commercialized high- $\mathrm{d}_{33}$ piezoelectric coefficient, hard ceramic plate (PZT 855, APC International Ltd.). A proven fast and economical approach, dicing, is used to fabricate freestanding PZT fins. Because no high-temperature process is involved, there is no thermal stress or shrinkage as might be present in more traditional 
processes such as sol-gel or sintering. A $20 \times 20 \times 1 \mathrm{~mm}$ PZT plate (2a) is mounted on a glass substrate using adhesive and diced into 150 micron-wide 'fins' with a spacing of 165 micron defined by the thickness of the diamond blade used (2b). The diced PZT plate is transferred to a second PDMS-coated glass slide and remains on the glass slide for the rest of process. A thick epoxy-based negative photoresist SU-8 (SU-8 2025, Microchem Co.) is then cast over the diced PZT plate. SU-8 pillars for electrical isolation are formed between the fins in alternating gaps by photolithography (2c). The pillar-to-pillar separation distance is $3.5 \mathrm{~mm}$. A double-layer (50nm-thick titanium and 2 micron-thick copper) is deposited on the PZT structures as well as the SU-8 isolation pillars. The thickness of the copper layer on the sidewall ranges from approximately $100 \mathrm{~nm}$ to 2 micron due to the nonuniform coating of the sputtered metal in the deep trenches. A silver-PMMA (40 vol\% Ag) ECPC blend is prepared and cast into the gaps between the PZT fins, and then vacuum-cured at $65^{\circ} \mathrm{C}$ (2d). After the excess polymer and metal on top of the PZT are polished away, the sample is diced into separate devices with any desired number of layers (2e). Multilayer devices, e.g, 10-layer devices with a dimension of $4 \times 3 \times 1 \mathrm{~mm}$ are then released from the substrate. Electrical leads are applied on both the left and right sidewalls. The devices are then poled by applying a $2 \mathrm{kV} / \mathrm{mm}$ electric field in the transverse direction of the layer (2f) at room temperature.

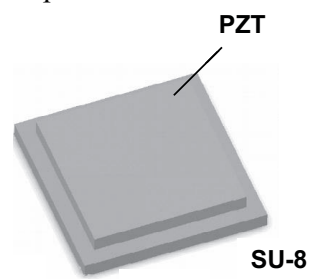

(a)

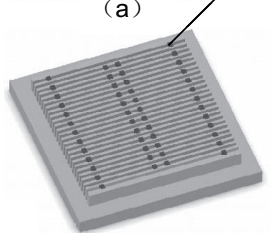

(c)

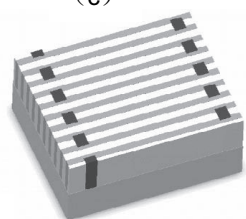

(e)

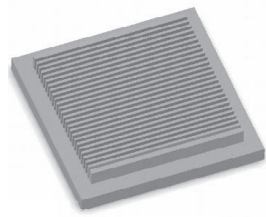

(b)

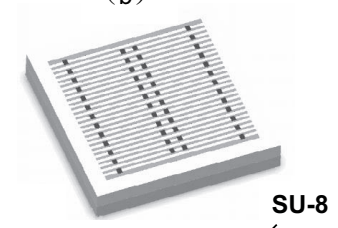

(d)

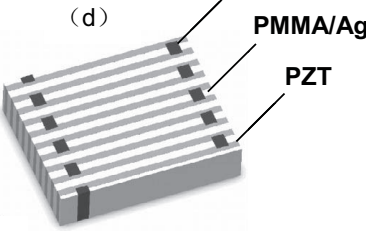

(f)
Figure 2. Fabrication process

SEM micrographs of diced PZT fins with SU-8 pillars are shown in Figure 3. The top view shows the uniform cuts with a PZT width of 150 micron and a spacing of 165 micron. Viewed from an angle, the SU-8 connects are found to be quite wellleveled with the PZT fins, with a tolerance of approximately $2 \%$ over the $1 \mathrm{~mm}$-tall SU-8 pillar.

However, the SU-8 pillars are tapered down from the top to the bottom as shown in Figure 3(b) and Figure 4(a). It is attributed in part to non-uniform SU-8 crosslinking over the high-aspect-ratio pillars (1:7) resulting from residual solvent gradient in the thick pillar and optical dose variation between the top and bottom portions. Because the optical exposure is from the top side, the top layer is overexposed and the bottom layer is relatively underexposed, which results in the variation of the lateral dimensions of the developed SU-8 structures. The same situation applies to the back-side exposure as illustrated in Figure 4(b). The undesired gaps between SU-8 and PZT could lead to electrode shorting.

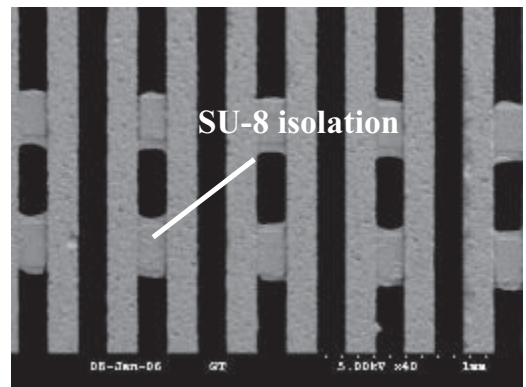

(a)

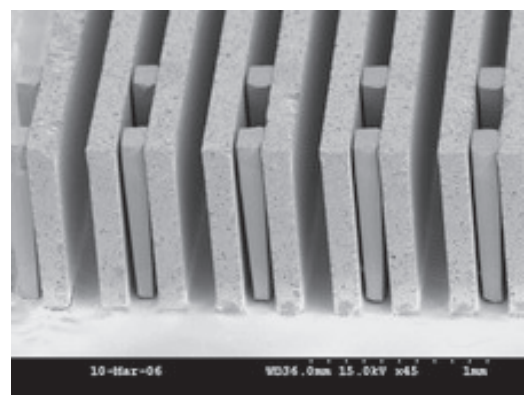

(b)

Figure 3. SEM images of diced PZT fins with SU-8 pillars for electrode isolation: (a) top view, (b) oblique view
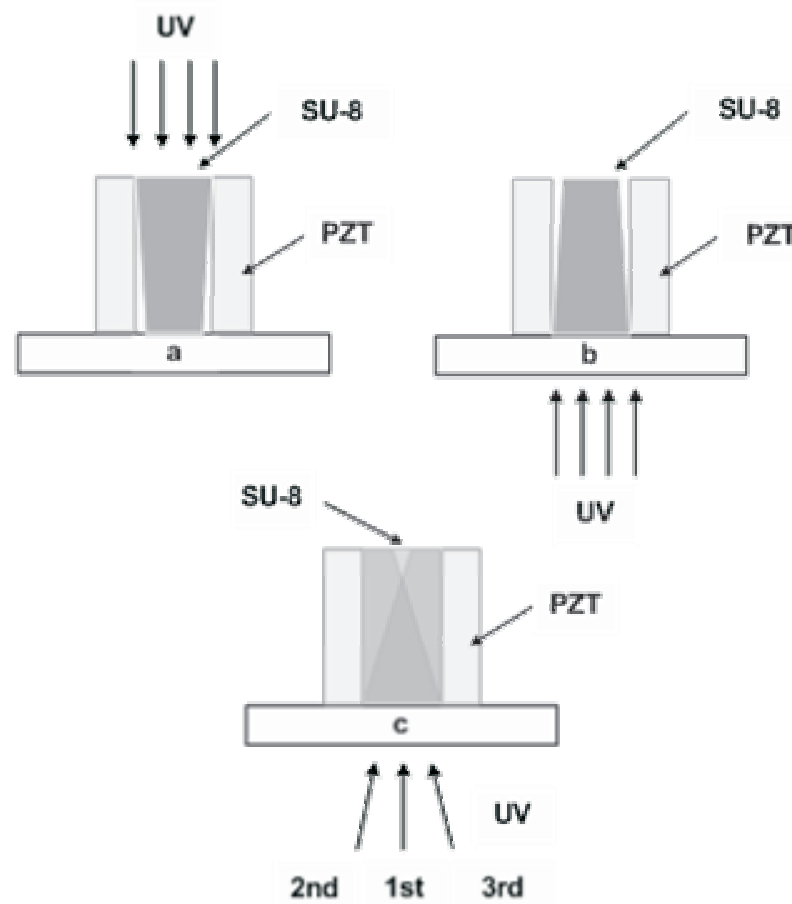

Figure 4. (a)Tapered SU-8 pillar formed from front-side exposure, (b) tapered SU-8 pillar formed from back-side exposure and (c) back-side inclined exposure scheme for fabrication SU-8 isolation pillars with conformal contact 
An improved method of photo-patterning SU-8 electrical isolation pillar structures is through back-side inclined exposure as shown in Figure 4(c) [6]. Schematic views of the process itself as well as the comparison with front-side and back-side exposures are shown in Figure 4. The experiment is started with deposition and patterning of 50nm-thick titanium and $200 \mathrm{~nm}$-thick copper on top of a glass substrate. The metal pre-patterning is utilized for pillar definition using back-side exposure. A layer of 20 micron-thick SU-8 2010 is spin-coated as an adhesive layer and baked at $65^{\circ} \mathrm{C}$ for $5 \mathrm{~min}$ and then at $95^{\circ} \mathrm{C}$ for $15 \mathrm{~min}$ on a hotplate. A PZT plate $20 \times 20 \times 1 \mathrm{~mm}$ in size is mounted on the prebaked SU-8 layer, and baked at $150^{\circ} \mathrm{C}$ for 6 hours to bond two pieces together through thermally crosslinking SU-8. The PZT plate is diced into 150 micron wide 'fins' with a spacing of 165 microns in alignment with the pre-patterned substrate. This substrate is subsequently exposed from the back vertically (i.e. at an incident angle of $90^{\circ}$ from the substrate), then exposed at $95^{\circ}$ and $85^{\circ}$ incident angles. SEM micrographs of diced PZT fins with SU-8 pillars formed by back-side exposure are shown in Figure 5. The magnified image in Figure 5 shows the desired conformal contact between SU-8 pillars and the sidewall of PZT layer.

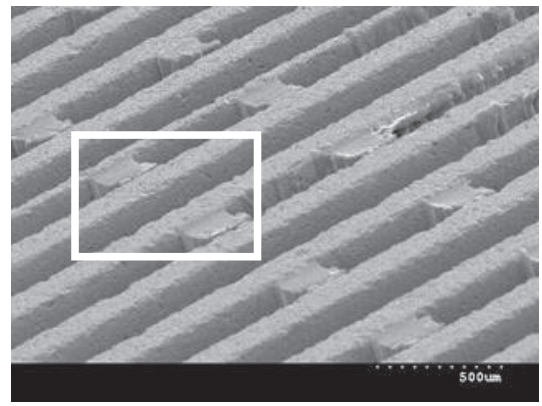

(a)

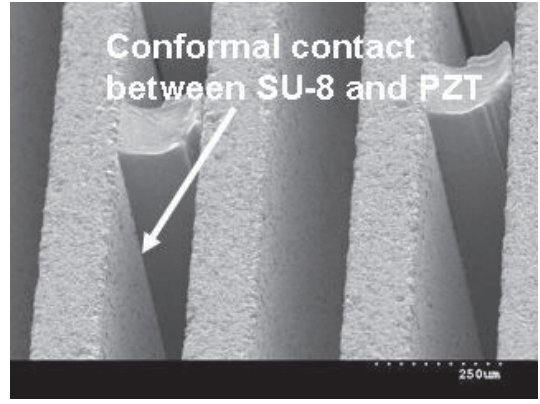

(b)

Figure 5. (a) SEM images of diced PZT fins with SU-8 pillars formed by back-side inclined exposure, (b) magnified image of two SU-8 pillars

\section{CHARACTERIZATION}

Before the testing of the multilayer piezoelectric actuators, the conductive polymer composite is first tested for its effectiveness as an electrode material for the PZT actuator. Thin slabs of PZT with a thickness of 150 micron and a lateral dimension of $20 \mathrm{~mm} \times 1 \mathrm{~mm}$ are prepared. Silver-PMMA ECPC (40 vol\% Ag) blend is applied on both sides of a single slab of PZT. For comparison purposes, PZT thin slabs with sputtered metal electrodes and with ECPC electrodes on top of sputtered metal electrodes are prepared as well. The property of ferroelectric polarization versus electric field (P-E) of each type is measured at
$0.1 \mathrm{~Hz}$ using a Sawyer-Tower circuit [7] at room temperature. The $\mathrm{P}-\mathrm{E}$ property is measured under an unpoled condition in all three cases.

Figure 6 shows the P-E hysteresis loops of a single PZT slab with different electrodes. For a desired remanent polarization value $\left(\mathrm{P}_{\mathrm{r}}\right)$ of $0.25 \mathrm{C} / \mathrm{m}^{2}$, the electric fields required for ECPC-only, ECPC on sputtered metal, and sputtered metal-only electrodes are $3.7 \mathrm{kV} / \mathrm{mm}, 2 \mathrm{kV} / \mathrm{mm}$, and $1.35 \mathrm{kV} / \mathrm{mm}$, respectively. The sputtered metal structures help reduce the required electric field and are favorable for low voltage applications. Accordingly, a sputtering step should be added before the molding the ECPC electrodes in the multilayer fabrication process.

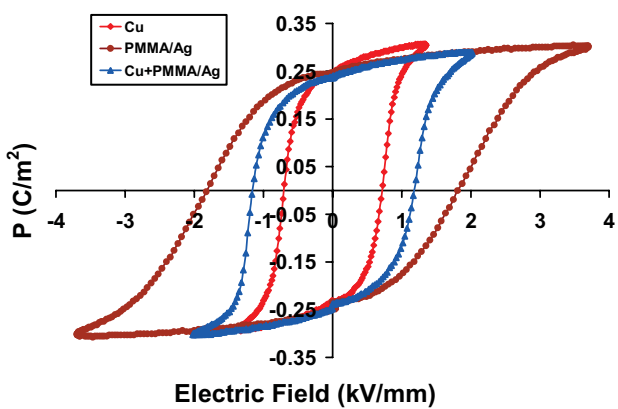

Figure 6. $P-E$ hysteresis loop for a single PZT layer with three types of electrodes

Laterally-stacked actuators with 8 and 10 layers have been successfully fabricated. Fig. 7(a) and 7(b) show a 10-layer and a part of an 8-layer stack actuator, respectively. Figure 8 shows the P-E hysteresis loops of a single PZT slab and of an 8-layer actuator with the same type of electrodes.

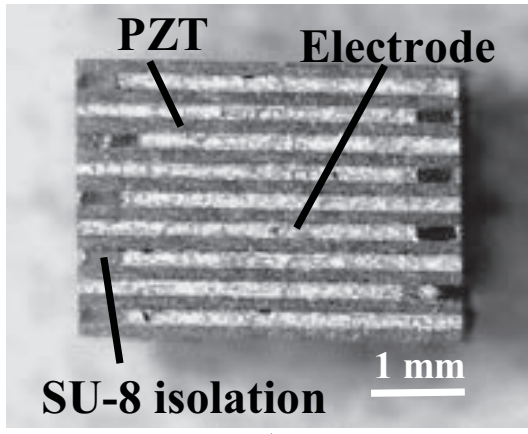

(a)

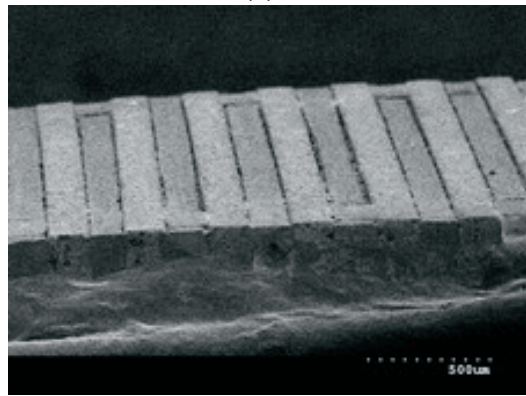

(b)

Figure 7. (a) Optical microscopy image of a 10-layer PZT actuator with conductive polymer electrode, (b) SEM image of an 8-layer PZT actuator (Note a probe wire has been attached) 


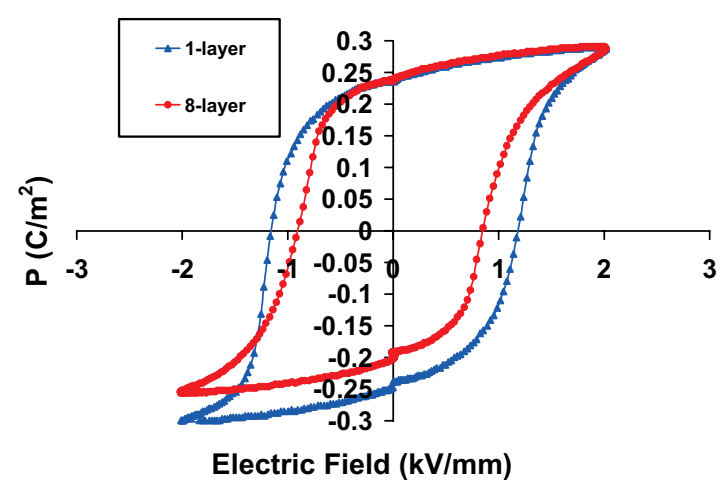

Figure 8. P-E hysteresis loop for a single PZT layer and an 8layer actuator

As shown in Figures 8, the multilayer PZT actuator has a hysterisis loop similar in shape to that of a single layer structure, with the former having a lower coercive field value $(0.84 \mathrm{kV} / \mathrm{mm})$ than the latter $(1.17 \mathrm{kV} / \mathrm{mm})$, which indicates better polymer electrode coverage on the sidewall of the multilayer actuator. The multilayer PZT actuator also exhibits a slightly asymmetric hysteresis behavior.

Finite element simulation is carried out to obtain the displacement of an 8-layer actuator as a function of applied electric field using ANSYS. The simulated displacement result for an 8 -layer actuator at a driving voltage of $100 \mathrm{~V}$ is 0.505 micron as shown in Figure 9. Experimental results for actuator displacement are measured using a laser displacement sensor (LK-G32, Kenyence Co.) under quasistatic conditions $(0.5 \mathrm{~Hz})$. Figure 10 illustrates the FEM results and experimental results of the top layer displacement of the actuator as a function of electric field for an 8layer device. The experimental results for displacement exhibit obvious linearity and agree reasonably well with the simulation result. The discrepancy may have resulted from the difference between the $d_{33}$ used in the simulation, which is a manufacturerspecified $d_{33}$ value of $630 \times 10^{-12} \mathrm{~m} / \mathrm{V}$, and the actual $\mathrm{d}_{33}$ value of the PZT layer after repoling the PZT in a transverse direction from the original polarization. The calculated $d_{33}$ value from the displacement measurement is $787 \times 10^{-12} \mathrm{~m} / \mathrm{V}$.

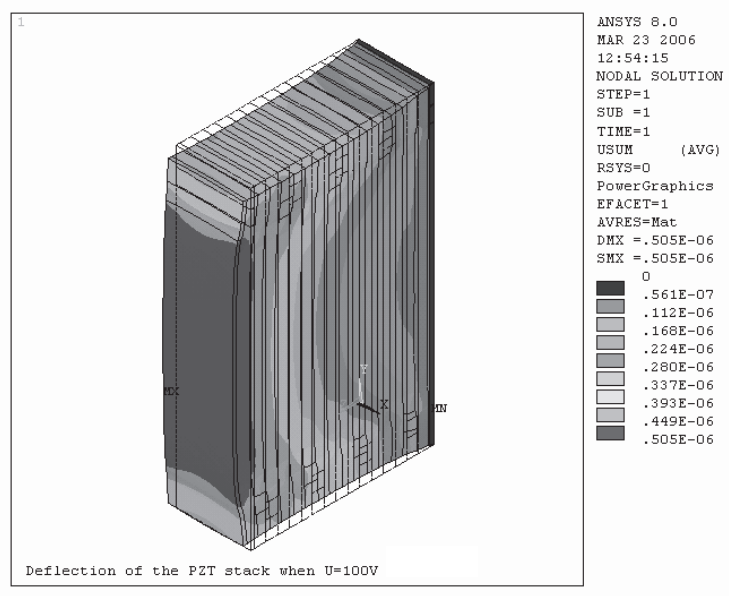

Figure 9. FEM simulation result for an 8-layer actuator at a driving voltage of $100 \mathrm{~V}$

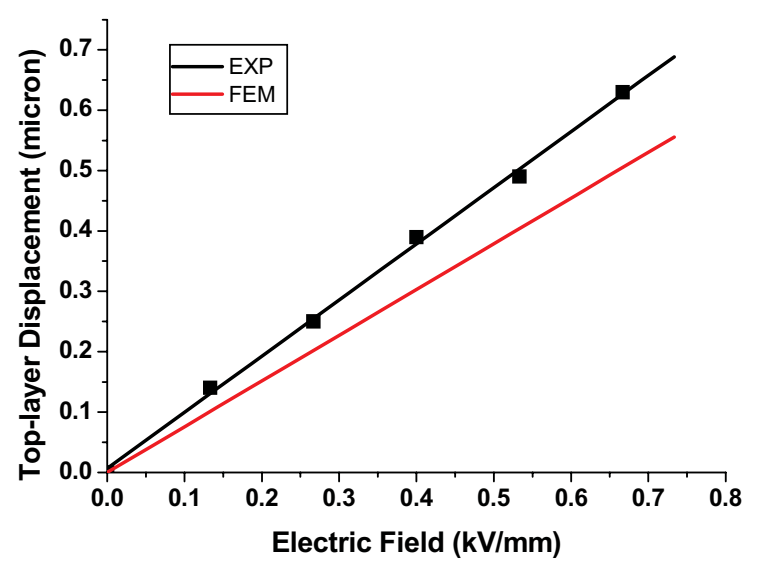

Figure 10. Top layer displacement of an 8-layer actuator as a function of applied electric field (maximum field of $0.66 \mathrm{kV} / \mathrm{mm}$ achieved at a voltage of $100 \mathrm{~V}$ )

\section{CONCLUSIONS}

Laterally-stacked multilayer PZT actuators have been fabricated using diced PZT multilayer, high aspect ratio SU-8 photolithography and molding of electrically conductive polymer composite electrodes. This fabrication process is simple and straightforward compared to previous lateral lamination approaches. An 8-layer device has shown a displacement of 0.63 micron at $100 \mathrm{~V}$ driving voltage, agreeing reasonably well with simulation results. The results indicate that the fabrication process of lateral lamination provides a valuable alternative for making compact, low-voltage multilayer piezoelectric micro-actuators. The fabricated PZT structures may be suitable for applications in microfluidics (as a microvalve or micropump actuator) and in optical zooming of camera lens.

\section{REFERENCES}

[1] S.Kawakita, T.Isogai, "Multi-layered piezoelectric bimorph actuator", 1997 International Symposium on Micromechanics and Human Science, 1997, pp. 73-78

[2] K. Yao, K.Uchino, et al., "Compact Piezoelectric Stacked Actuators for High Power Applications" IEEE Transactions on Ultrasonics, Ferroelectrics and Frequency Control, 47(4), July 2000, pp.819 - 825

[3] T. Lilliehorn and S. Johansson, "Fabrication of multilayer 2D ultrasonic transducer microarrays by green machining" $J$. icromech. Microeng. 14 (2004), pp.702-709

[4] W. Zhu, K. Yao, Z. Zhang, "Design and fabrication of a novel piezoelectric multilayer actuator by thick-film screen printing technology", Sensors and Actuators, 86 (2000), pp.149-153

[5] G. Suzuki and M. Esashi, "Planer Fabrication of Multilayer Piezoelectric actuator by groove cutting and electroplating",Proc. MEMS 2000, pp. 46-51

[6] Y.-K. Yoon, J.-H. Park, and M.G. Allen, "Multidirectional UV lithography for complex 3-D MEMS structures," J. MEMS, 2006 (in press)

[7] C. B. Sawer and C. H. Tower, "Rochelle salt as a dielectric", Physical review, 35(1930), pp.269-273 